\title{
Amygdala volumetry in "imaging-negative" temporal lobe epilepsy
}

\author{
S P C Bower, S J Vogrin, K Morris, I Cox, M Murphy, C J Kilpatrick, M J Cook
}

J Neurol Neurosurg Psychiatry 2003;74:1245-1249

See end of article for authors' affiliations

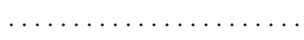

Correspondence to: Dr S P C Bower, Monash Institute of Neurological Diseases, 246 Clayton Road, Clayton, Victoria, Australia 3168

s.bower@

southernhealth.org.au

Received

24 October 2002

Accepted in revised form

4 March 2003

\begin{abstract}
Objective: Although amygdala abnormalities are sometimes suspected in "imaging-negative" patients with video EEG confirmed unilateral focal epilepsy suggestive of temporal lobe epilepsy (TLE), amygdala asymmetry is difficult to assess visually. This study examined a group of "imaging-negative" TLE patients, estimating amygdala volumes, to determine whether cryptic amygdala lesions might be detected.

Methods: Review of video EEG monitoring data yielded 11 patients with EEG lateralised TLE and normal structural imaging. Amygdala volumes were estimated in this group, in 77 patients with pathologically verified hippocampal sclerosis (HS), and in 77 controls.

Results: Seven of 11 "imaging-negative" cases had both significant amygdala asymmetry and amygdala enlargement, concordant with seizure lateralisation. Although significant amygdala asymmetry occurred in 35 of 77 HS patients, it was never attributable to an abnormally large ipsilateral amygdala. Compared with patients with HS, patients with amygdala enlargement were less likely to have suffered secondarily generalised seizures $(p<0.05)$, and had an older age of seizure onset $(\mathrm{p}<0.01)$.

Conclusion: Abnormal amygdala enlargement is reported in seven cases of "imaging-negative" TLE. Such abnormalities are not observed in patients with HS. It is postulated that amygdala enlargement may be attributable to a developmental abnormality or low grade tumour. It is suggested that amygdala volumetry is indicated in the investigation and diagnosis of "imaging-negative" TLE.
\end{abstract}

$\mathrm{H}^{\prime}$ ppocampal sclerosis (HS) is the most common abnormality associated with temporal lobe epilepsy (TLE) accounting for about $70 \%$ of cases in surgical series. ${ }^{1} \mathrm{~A}$ number of studies have shown this abnormality is reliably detected by magnetic resonance imaging (MRI). ${ }^{2}$ Foreign tissue lesions account for most of the remainder, but there exists a small group who are "imaging-negative". In TLE associated with HS the amygdala is an important source of seizures as demonstrated by invasive electrophysiological studies. ${ }^{5}$ Isolated amygdala sclerosis has been described ${ }^{67}$ and temporal lobe tumours most commonly involve the amygdaloid region. ${ }^{8}$ Such lesions however are often not readily identifiable on routine MRI. ${ }^{6}{ }^{9}$ Although hippocampal volumetry is now an established technique permitting detection of subtle degrees of hippocampal atrophy, ${ }^{10}$ there have been few amygdala volumetric studies, no doubt partly because of the amygdala's poorly demarcated anatomical boundaries. ${ }^{11-13}$

We have encountered several cases where amygdala enlargement was suspected on visual inspection of routine MR images. Given difficulties encountered in visually estimating the volume of this structure, we performed a volumetric study of "imaging-negative" TLE patients. As others have raised the possibility of isolated amygdaloid sclerosis in TLE, ${ }^{6}$ we also examined "imaging-negative" patients to determine whether this was an alternative pathology. Given that amygdaloid sclerosis is reported in association with HS we performed similar measures in a group with pathologically verified HS.

\section{METHODS}

We reviewed the video EEG monitoring data of the Comprehensive Epilepsy Program at St Vincent's Hospital and Royal Melbourne Hospital between 1993 and 1997. There were 174 cases who had undergone presurgical evaluation, and who after video EEG monitoring, had lateralised seizures thought clinically and electrically consistent with an origin in the temporal lobe. The original MR images of all patients were reviewed. Careful inspection of 2D and 3D reformatted images was performed to exclude small neocortical lesions. "Imagingnegative" was defined by the absence of a neocortical lesion, normal hippocampal volumetry, and absence of any increased signal in the mesial temporal lobes on routine visual assessment. From these 174 patients we identified 11 patients (four women, seven men) with video EEG confirmed unilateral focal epilepsy suggestive of TLE who were "imaging-negative".

There were 77 patients (46 women, 31 men) who underwent temporal lobectomy and had pathologically verified HS. These 77 patients formed our HS group. The controls were 77 age and sex matched volunteers (46 women, 31 men) who completed a questionnaire excluding past head injury, medical or psychiatric history.

In all patients the following clinical details were recorded prospectively on admission for video EEG monitoring: age of onset of habitual epilepsy, history of febrile convulsions, frequency of complex partial seizures during the year preceding imaging, and lifetime occurrence of secondarily generalised seizures. Numbers of left handed persons were too small to permit a meaningful comparison of the effect of handedness in either patients or controls.

All controls had a coronal Tl weighted 3D spoiled gradient echo (SGPR) sequence, 9.7/4/1 (repetition time/echo time/ excitations), at $1.5 \mathrm{~mm}$ thickness with no interslice gap. The flip angle was 20 degrees, matrix size $256 \times 192$, field of view $200 \times 150 \mathrm{~mm}$, with resultant voxel dimensions $0.78 \times 0.78 \times 1.50$ $\mathrm{mm}$.

Abbreviations: TLE, temporal lobe epilepsy; HS, hippocampal sclerosis; $M R I$, magnetic resonance imaging; $A M V$, amygdala volume; $A V R$, amygdala volume ratio 

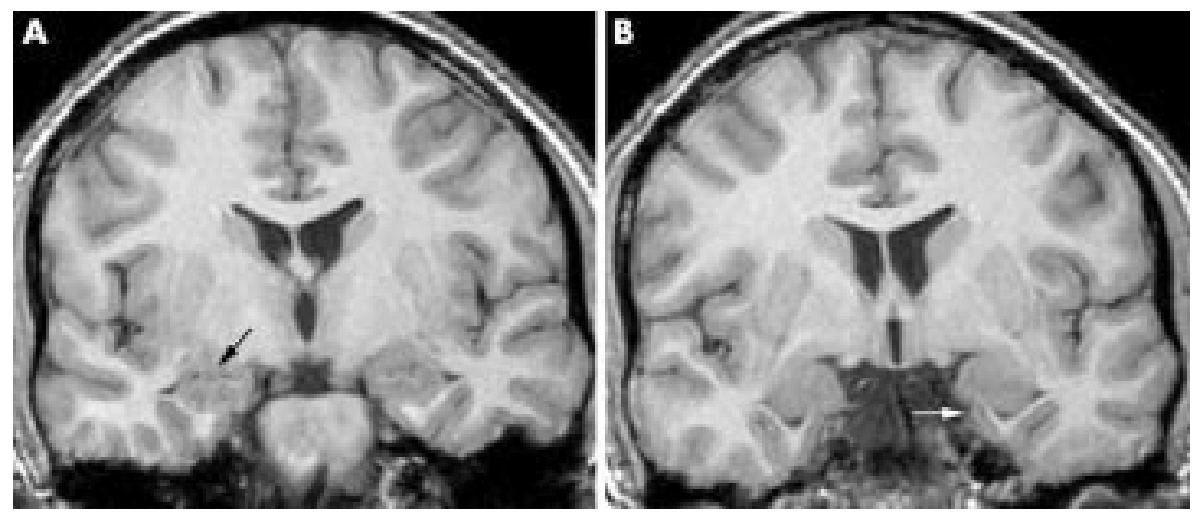

Figure 1 (A) Black arrowhead marks the alveus separating the amygdala from the hippocampal head. (B) White arrowhead marks the tentorial indentation and the inferomedial boundary of the amygdala.
The same 124 slice coronal volumetric sequence was performed on all patients admitted for video EEG monitoring. In addition, axial T2 (2646/98/2), axial proton density $(2864 / 16 / 2)$, coronal T2 $(2864 / 80 / 1)$, coronal proton density $(2864 / 20 / 1)$, and coronal inversion recovery $(6335 / 0 / 2)$ sequences were obtained.

MR images were transferred off line to an IBM RISC 6000 UNIX workstation using ANALYZE 7.55 (Mayo Foundation, Minnesota, 1995). All scans were reformatted to correct for tilt, so that the lateral fissures and greatest length of fornix were as symmetrical as possible. Hippocampal and amygdala volumes were measured according to established techniques and with reference to an anatomical atlas. ${ }^{1014}$ Volumetric measurements were performed retrospectively by SPCB from original images, but with the knowledge that the scan was either a control or a patient. Thresholding was used to define the more indistinct grey-white boundaries of the amygdaloid complex that was then measured slice by slice in a caudorostral direction. There were no technical difficulties in outlining the amygdaloid complex in any patient or control.

\section{Amygdala volumetric protocol}

The first slice of amygdala was taken as that slice in which the uncinate gyrus was continuous over the inferior horn of the lateral ventricle and the amygdala was separated from hippocampus at its narrowest point. More anteriorly the grey-white boundary was traced to separate amygdala from substantia innominata, inferior putamen, and claustrum. The alveus separated the amygdala from the hippocampal head and with careful attention to previous and forthcoming slices, the alveus could be reliably identified in our $1.5 \mathrm{~mm}$ slices (fig 1A). The most anterior slice was the opening of the endorhinal sulcus to form the lateral fissure. Ambient gyrus formed the medial border, and the tentorial notch marked the inferior extent of the overlying enterorhinal cortex (fig 1B). If this notch was not easily seen, a straight line was drawn from the inferior and medial border of the amygdala within the temporal white matter. Inferior horn of lateral ventricle marked the lateral border.

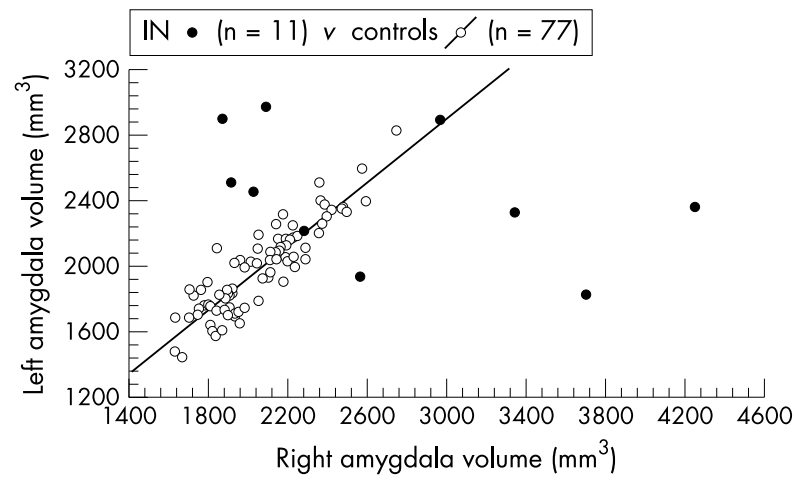

Figure 2 Amygdala volumes in "imaging-negative" TLE.
Amygdala volumes (AMV) were calculated by summing cross sectional areas and multiplying by $1.5 \mathrm{~mm}$ slice thickness (Cavalieri's principle). On average AMV was estimated from 10 slices. The normal control range was defined as mean (2SD). Symmetry was expressed as a ratio of right over left volumes, amygdala volume ratio (AVR), and a similar reference range of mean (2SD) was used.

Intra-rater repeatability was assessed bilaterally in 50 randomly selected controls. The mean intra-rater difference between the first and repeat measurement was $+2.0 \mathrm{~mm}^{3}$ (range $-263 \mathrm{~mm}^{3}$ to $+342 \mathrm{~mm}^{3}$, SD $135 \mathrm{~mm}^{3}$ ). The coefficient of repeatability is defined as 2 SD divided by the mean of the average measurements and equals $13.0 \%{ }^{16}$ Inter-rater coefficient of repeatability was estimated in 20 randomly selected controls and equals $14.5 \%$. The Student's $t$ test, Fisher's exact test, and Mann-Whitney $U$ tests were used for statistical analyses.

\section{RESULTS}

Amygdala volumes in the 77 controls were normally distributed. All volumes were expressed in cubic millimetres $\left(\mathrm{mm}^{3}\right)$. The mean total amygdala volume in men was 4294 (485) $\mathrm{mm}^{3}$ compared with $3890(459) \mathrm{mm}^{3}$ in women $(t=3.7$, $\mathrm{p}=0.0004)$. The right and left amygdala volumes were not statistically significantly different in men $\left(2180(237) \mathrm{mm}^{3} \mathrm{com}-\right.$ pared with $2113(262) \mathrm{mm}^{3}$ ) or women (1988 (239) $\mathrm{mm}^{3}$ compared with $\left.1902(245) \mathrm{mm}^{3}\right)$. Using the criteria for normal ranges defined above, the normal range for amygdala volumes in men was $1650 \mathrm{~mm}-2650 \mathrm{~mm}^{3}$, and in women 1470 $\mathrm{mm}-2420 \mathrm{~mm}^{3}$. The normal amygdala volume ratio was slightly larger for women 1.05 (0.07) (0.91-1.19), than men, 1.04 (0.06) (0.92-1.16).

Table 1 shows the amygdala volumes and amygdala volume ratios in the 11 "imaging-negative" patients. There were seven

Table 1 Clinical features and amygdala volumes in 11 "imaging-negative" cases. Abnormal volumes and ratios in bold italics

\begin{tabular}{llllll}
\hline Sex, age & $\begin{array}{l}\text { Seizure } \\
\text { onset }(y)\end{array}$ & $\begin{array}{l}\text { EEG } \\
\text { focus }\end{array}$ & RAV & LAV & AVR \\
\hline $\mathrm{m}, 29$ & 9 & $\mathrm{R}$ & $\mathbf{3 7 1 6}$ & 1818 & $\mathbf{2 . 0 4}$ \\
$\mathrm{m}, 60$ & 58 & $\mathrm{R}$ & $\mathbf{4 2 6 1}$ & 2352 & $\mathbf{1 . 8 1}$ \\
$\mathrm{m}, 30$ & 14 & $\mathrm{~L}$ & 2102 & $\mathbf{2 9 6 5}$ & $\mathbf{0 . 7 1}$ \\
$\mathrm{m}, 34$ & 24 & $\mathrm{~L}$ & 2044 & 2455 & $\mathbf{0 . 8 3}$ \\
$\mathrm{m}, 32$ & 25 & $\mathrm{~L}$ & 1906 & 1744 & 1.09 \\
$\mathrm{~m}, 19$ & 14 & $\mathrm{R}$ & $\mathbf{2 9 7 5}$ & $\mathbf{2 8 8 4}$ & 1.03 \\
$\mathrm{~m}, 20$ & 14 & $\mathrm{R}$ & 2290 & 2217 & 1.03 \\
$\mathrm{f}, 63$ & 62 & $\mathrm{~L}$ & 1877 & $\mathbf{2 8 8 9}$ & $\mathbf{0 . 6 5}$ \\
$\mathrm{f}, 35$ & 34 & $\mathrm{R}$ & $\mathbf{3 3 5 2}$ & 2315 & $\mathbf{1 . 4 5}$ \\
$\mathrm{f}, 30$ & 16 & $\mathrm{R}$ & $\mathbf{2 5 7 2}$ & 1932 & $\mathbf{1 . 3 3}$ \\
$\mathrm{f}, 37$ & 16 & $\mathrm{~L}$ & 1925 & $\mathbf{2 5 0 4}$ & $\mathbf{0 . 7 7}$ \\
\hline
\end{tabular}

$L A V$, left amygdala volume $\left(\mathrm{mm}^{3}\right) ; R A V$, right amygdala volume $\left(\mathrm{mm}^{3}\right)$; AVR, amygdala volume ratio (RAV/LAV). 


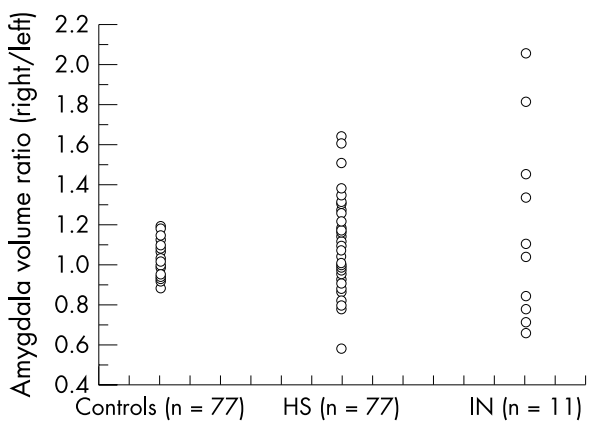

Figure 3 Amygdala volume ratios in controls, hippocampal sclerosis patients (HS), and "imaging-negative" patients (IN).

patients with both abnormal volume ratios and absolute volumes outside the normal range as indicated in italics. All seven patients had amygdala enlargement ipsilateral to the ictal focus. No cases of amygdala atrophy were identified. Figure 2 is a scatter plot of right compared with left amygdala volumes in these 11 "imaging-negative" patients, compared with the 77 controls. There were seven cases, clearly separated from controls, with both significant asymmetry and absolute volumes above the normal control range.

Figure 3 shows the amygdala volume ratios for the three groups: normal controls $(\mathrm{n}=77)$, patients with verified hippocampal sclerosis (HS, $\mathrm{n}=77$ ), and "imaging-negative patients" (IN, $\mathrm{n}=11)$. Although 35 patients in the HS group had abnormal amygdala ratios, it was either attributable to ipsilateral amygdala atrophy $(n=15)$, contralateral amygdala atrophy $(n=5)$, or asymmetry, but with volumes within the normal range $(n=15)$. Of note amygdala asymmetry was never attributable to ipsilateral amygdala enlargement in patients with confirmed HS.
Table 2 Clinical features of patients with amygdala enlargement $(\mathrm{AE})$ compared with patients with verified hippocampal sclerosis (HS)

\begin{tabular}{llll}
\hline & AE $(n=7)$ & HS $(n=77)$ & p Value \\
\hline Febrile seizures & $1(14 \%)$ & $37(48 \%)$ & 0.12 \\
Ever generalised seizures & $2(29 \%)$ & $7(85 \%)$ & $0.01^{*}$ \\
Mean age of onset $(y)$ & $29(14-62)$ & $11(0.5-38)$ & $0.01 \dagger$ \\
\hline *Fisher's exact test; †Mann-Whitney U test. & & \\
\hline
\end{tabular}

Table 2 compares the clinical details of the seven patients with amygdala enlargement (AE) versus the 77 patients with confirmed HS. There was a trend towards fewer febrile convulsions in the AE group, statistically significantly fewer generalised seizures $(p=0.01)$, and an older mean age of seizure onset $(p=0.01)$.

Of the 11 imaging-negative cases only one proceeded to surgery, all others were controlled medically. MR images from two cases are shown. Figures 4A and 4B show a left amygdala mass in a 62 year old woman with an eight month history of seizures, starting abruptly and occurring up to 12 times daily. She reported an "electric shock sensation" in both legs with accompanying piloerection, epigastric sensation, hand fidgeting, and often confusion lasting only minutes. Left sided seizures were recorded and she responded to carbamazepine.

Figures 5A and 5B show a right amygdala mass in a 29 year old man with a 20 year history of frequent complex partial seizures. He reported a "funny sick" feeling often precipitated by an amusing situation. Febrile convulsions occurred in childhood. These gelastic seizures were captured on video EEG and a right sided anterior temporal lobe focus was recorded. Surgery was performed in this single case because of poor seizure control. Pathology confirmed a glioneuronal hamartoma.
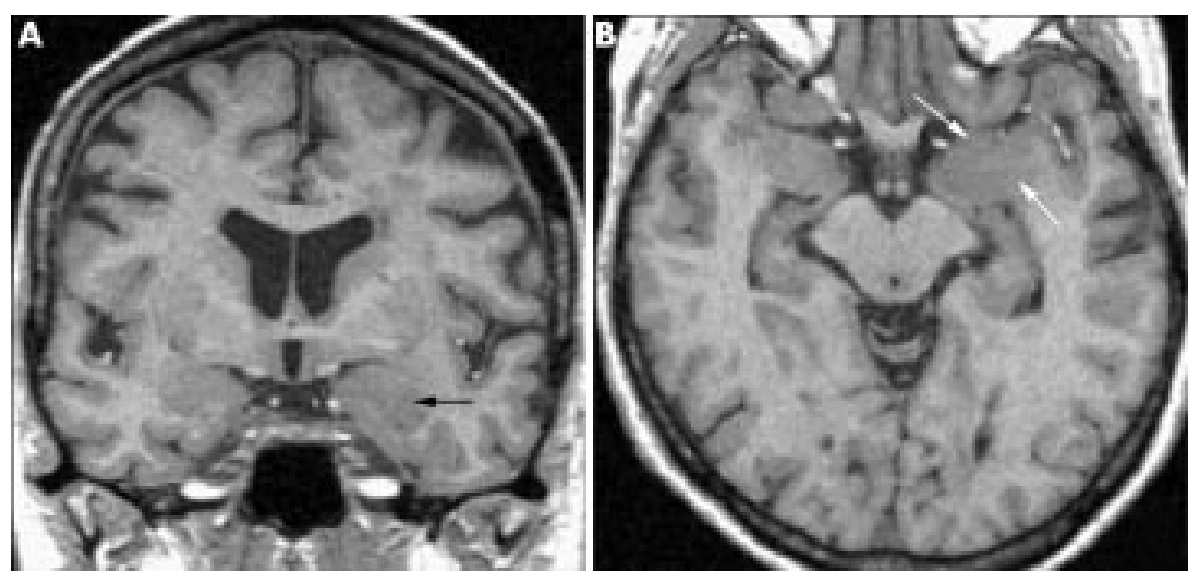

Figure 4 (A) T1 weighted coronal MR image shows left amygdala mass (black arrow) in a 63 year old woman (amygdala volume $2889 \mathrm{~mm}^{3}$ normal range $<1913 \mathrm{~mm}^{3}$ ). (B) Same patient in axial plane, with left amygdala mass (between white arrows).
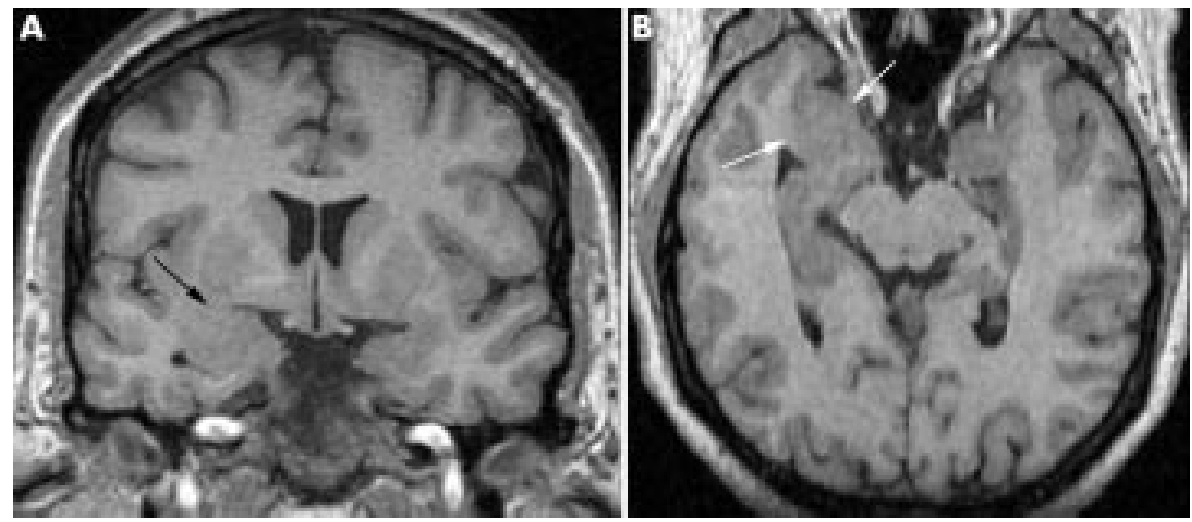

Figure 5 (A) T1 weighted coronal MR image shows right amygdala mass (black arrow) in a 29 year old man, pathologically confirmed as glioneuronal hamartoma (amygdala volume $3716 \mathrm{~mm}^{3}$, normal range $<2255 \mathrm{~mm}^{3}$ ). (B) Same patient in axial plane, with right amygdala mass between white arrows. 
On review of coronal and axial T2 images there was subtle increased amygdala T2 signal in only one of seven cases of amygdala enlargement. The absence of increased $\mathrm{T} 2$ signal no doubt contributed to the difficulty in recognising amygdala enlargement on visual inspection of routine MR images.

Pathological examination of the amygdala in temporal lobectomy specimens was performed in 16 consecutive patients with HS. All but one patient had abnormal glial fibrillary acidic protein (GFAP) staining, indicative of amygdaloid gliosis.

\section{DISCUSSION}

There have been no previous studies estimating amygdala volumes in "imaging-negative" TLE. We found seven patients with unexpected amygdala enlargement in 11 "imagingnegative" patients. No cases of isolated amygdala atrophy were found. None of these cases had been recognised by experienced neuroradiologists routinely reporting films. Subtle increased T2 signal was present on retrospective review of MR images in only one patient. We postulate that these cases of abnormal amygdala asymmetry and enlargement may represent a developmental abnormality, or the "alien tissue lesions" that Bruton noted "occurred more often in the amygdaloid region". ${ }^{8}$ In his neuropathological review of 249 temporal lobectomies there were eight astrocytic glial lesions, each forming a discrete mass, and four of these specifically involved the amygdala. Of other cell types, the amygdala was involved in five of six oligodendroglial lesions, 6 of 13 mixed glial lesions, and six of nine neuronoglial lesions. Bruton also noted "the extremely common involvement of the amygdaloid region in the patients with sclerosis of the Ammon's horn."

But despite this known pathology, recognition of amygdala abnormalities on routine MR images has been difficult. Hudson et al found only a small proportion of patients with isolated histologically confirmed amygdaloid sclerosis had MR images consistent with temporal lobe atrophy. ${ }^{6}$ Bronen et al reported amygdala atrophy in only $12 \%$ of patients with HS verified by histological analysis. ${ }^{9}$ As in our study hyperintense T2 signal was present in only an occasional patient.

Quantitative T2 relaxometry of the amygdala, or "amygdala T2 mapping" has been proposed as a more systematic method than volumetry in detecting amygdaloid lesions. Van Paesschen et al reported findings in 82 patients with intractable TLE and 20 controls subjects. ${ }^{4}$ An abnormally high T2 signal was present on the side of classic HS in 23 of 44 patients (52\%) with unilateral HS. Two of these patients with a high amygdala T2 signal had amygdala sclerosis confirmed pathologically. Of 31 patients with normal quantitative hippocampal measures, equivalent to our "imaging-negative" patients, 15 had an isolated abnormal amygdala T2 signal. Pathology was available in two cases confirming amygdala gliosis in one and microdysgenesis in another. The authors claim that atrophy is probably not a feature of most epileptogenic lesions of the amygdala, and that T2 mapping with FLAIR imaging might be a better technique than amygdala volumetry to detect these lesions. However, they did not compare their data with volumetry because of their poor inter-rater repeatability.

Based on our amygdala volumetric analysis of 77 patients with HS, ipsilateral amygdala atrophy is a comparatively infrequent accompaniment (19.5\%), and is almost as likely to be contralateral (15.6\%). However of 16 consecutive patients with HS where the amygdala was available for pathological examination, abnormal GFAP positivity (indicative of gliosis) was present in all but one. In keeping with previous pathological studies it is probable that amygdala gliosis frequently accompanies HS but does not usually lead to volume loss detectable by MR imaging. Our finding of almost universal amygdala gliosis alongside HS in temporal lobectomy specimens, leads us to query Van Paesschen's assumption that a high T2 signal is specific for amygdala gliosis.
On the other hand abnormally large amygdala volumes have been correlated with tumours.

Watson reported two pathologically verified cases of medial temporal lobe heterotopias, with increased hippocampal and amygdala volumes ipsilateral to electroencephalographic seizure origin. ${ }^{17}$ In one of our cases with an enlarged right amygdala pathology confirmed a glioneuronal hamartoma. The reason we do not have further pathological confirmation is that all other patients' seizures are currently well controlled on anticonvulsant medication, a dissimilar situation to the usual natural history of HS.

In all our patients seizures were lateralised to the hemisphere containing the enlarged amygdala. Importantly in two cases distortion of the head of the hippocampus by the enlarged amygdala might have suggested contralateral hippocampal atrophy, based on visual analysis. However, seizures were clearly lateralised to the hemisphere containing the larger amygdala, and volumetric assessment confirmed normal hippocampal volumes. This highlights the importance of considering abnormal amygdala enlargement when seizure localisation is discordant with apparent hippocampal atrophy.

In our cases the amygdala appears uniformly enlarged accounting for the measured asymmetry. In fact total amygdala volumes are probably underestimated, as in several cases the enlarged amygdala extends more anteriorly into the temporal lobe, outside our chosen anatomical boundary. Cases must be carefully reformatted to assess this aspect, so that the stem of the temporal lobe in the lateral fissure and the longest section of the fornix are as symmetrical as possible. Including the portion of the amygdala anterior to the lateral fissure might identify additional patients, but boundary definition in this region is difficult. If there is prominent grey matter extending medially, anterior to the lateral fissure, our findings suggest that radiologists consider the possibility of an ipsilateral larger amygdala. Viewing the scans axially often gives a more convincing impression of a bulky amygdala, (figs 4B and $5 \mathrm{~B})$ though again tilt may give a misleading impression of asymmetry, especially in standard $5 \mathrm{~mm}$ thick axial slices. This is another advantage of $3 \mathrm{D}$ volumetric imaging, compared with routine MR imaging, where reformatting in all planes may not be available.

In our "imaging-negative" group we found no examples of isolated amygdala atrophy. Previous reports of isolated amygdaloid sclerosis imply indirectly that this might be anticipated. Formal amygdala volumetry has not yet been reported in such a series. The concept of amygdaloid pathology in the absence of HS in TLE was proposed by Hudson. ${ }^{6}$ These patients had normal hippocampal neuronal counts, but abnormally low amygdala neuronal counts. However, the severity of amygdala neuronal loss was similar in the patients with isolated amygdaloid sclerosis compared with the patients with combined amygdaloid and hippocampal sclerosis. An alternative interpretation is that amygdala gliosis is a non-specific occurrence in the brains of most TLE patients, and when found in isolation does not signify a distinctive abnormality. In Wolf's pathological examination of 70 temporal lobectomy specimens there were no cases of isolated amygdaloid sclerosis, attesting to the rarity of such an entity. ${ }^{18}$

Previous authors studying the amygdala in TLE have not advocated the use of volumetry, because of an inter-rater repeatability of more than $20 \% .{ }^{4}{ }^{19}$ In our institution amygdala volumetry has an intra-rater and inter-rater coefficient of repeatability of $13.0 \%$ and $14.5 \%$, when using $1.5 \mathrm{~mm}$ slices and reformatting to correct for tilt. Expressed another way, our average inter-rater difference is $6.3 \%$ of mean value, which compares favourably with $9.0 \%$ reported in earlier studies. ${ }^{11}$ Our normal ranges and amygdala volume ratios are consistent with recently reported data. ${ }^{20}$

The frequency of "imaging-negative" TLE varies as would be expected with the methods used to define "imagingnegative" TLE. Rapid changes in MR techniques and increasing field strength of clinical MR scanners has resulted in a 
continuous decrease in the number of patients classified "imaging-negative" TLE. In Van Paesschen's quantitative MR study of the amygdala in TLE, $20 \%$ of patients were "imagingnegative", as defined by a normal routine MR image, normal hippocampal volumetry, and normal T2 mapping of both the hippocampus and amygdala. ${ }^{4}$ In pathological studies before MR imaging was available, no definite pathology to account for TLE was variously reported in between $4 \%$ to $20 \%$ of patients. ${ }^{21-23}$ We reviewed our video EEG monitoring data and found 11 cases where there was no clear MR imaging abnormality. Our monitored patients are not representative of the general population of temporal lobe epilepsy. Most are monitored because they have a MR abnormality and are being considered for surgery. Therefore we cannot estimate the true frequency of "imaging-negative" TLE from this study and do not claim that it is only 11 of 174 or $6.3 \%$. But in our practice, with detailed MR imaging, such cases are comparatively uncommon, and as we have found, may be associated with abnormal amygdala enlargement.

Possible sources of error were considered. The abnormally large amygdala might be at the upper limit of control ranges. By considering men and women separately, and a large number of controls, we have accounted for individual variation in brain size. ${ }^{24}$ It is possible that loss of grey-white differentiation associated with hippocampal or other temporal lobe pathology might cause an abnormally large amygdala volume measurement. The fact that we were able to define the edges of the amygdala in all patients with HS would make this unlikely. It is theoretically possible that acute seizure activity might cause brain oedema and amygdala enlargement, however none of the patients were scanned within 48 hours of seizures. As amygdala enlargement was not found in any of the patients with HS, it is unlikely any of these possible sources of error account for our findings.

"Imaging-negative" TLE is a difficult group to characterise and without intracranial electrode studies or postoperative data, we cannot be certain that all patients had TLE, as compared with other partial epilepsies. Although patients with occipital, parietal, and orbitofrontal foci can mimic the clinical features of TLE, given that the ictal EEG was well lateralised and the clinical features were consistent with TLE, we were reasonably confident of the diagnosis of TLE.

Clinically there were fewer febrile convulsions, an older age of onset, and less secondarily generalised seizures in patients with amygdala enlargement compared with those with HS. This is consistent with findings in Bruton's series of 249 temporal lobectomies. ${ }^{8}$ The group of "alien tissue lesions" uncommonly had a past history of febrile seizures and had an older age of seizure onset, compared with the group with Ammon's horn sclerosis, providing further support that amygdala enlargement correlates with amygdaloid tumour. It has been suggested that the abnormalites detected by amygdala T2 mapping and amygdala volumetry may not be identical. ${ }^{4}$ An alternative explanation is that so called amygdala gliosis, whether based on MR amygdala volumes or T2 mapping, is not a distinct entity. In contrast, the finding of an enlarged amygdala defines a subgroup of "imaging-negative" TLE patients that are clinically different to those with HS.

\section{ACKNOWLEDGEMENTS}

SPCB was supported by an epilepsy research fellowship from Reckitt and Colman.

\section{Contributors}

SPCB measured amygdala volumes in patients and controls, and collected clinical data. SJV and MJC measured hippocampal volumes in patients and controls. KM helped with image processing. IC reviewed all routine MR imaging. MM provided all amygdala surgical specimens. SPCB, CJK, and MJC took an active part in all aspects of the study and manuscript preparation.

\section{Authors' affiliations}

S P C Bower, S J Vogrin, K Morris, I Cox, M Murphy, M J Cook, Department of Clinical Neurosciences, St Vincent's Hospital, Melbourne, Australia

S P C Bower, C J Kilpatrick, Melbourne Neuroscience Centre, Royal Melbourne Hospital, Melbourne, Australia

C J Kilpatrick, Department of Medicine, Royal Melbourne Hospital

\section{REFERENCES}

1 Babb TL, Pretorius JK. Pathological substrates of epilepsy. In: Wylie E, ed. The treatment of epilepsy: principles and practice. Philadelphia: Lea and Febiger, 1993:55-70

2 Berkovic SF, Andermann F, Olivier A, et al. Hippocampal sclerosis in temporal lobe epilepsy demonstrated by magnetic resonance imaging. Ann Neurol 1991;29:175-82.

3 Cascino GD, Jack C, Parisi JE, et al. Magnetic resonance imaging-based volume studies in temporal lobe epilepsy: pathological correlations. Ann Neurol 1991;30:31-6.

4 Van Paesschen W, Connelly A, Johnson CL, et al. The amygdala and intractable temporal lobe epilepsy: a quantitative magnetic resonance imaging study. Neurology 1996;47:1021-31.

5 King D. Topographic distribution of seizure onset and hippocampal atrophy: relationship between MRI and depth EEG. Electroencephalogr Clin Neurophysiol 1997; 103:692-7.

6 Hudson LP, Munoz DG, Miller L, et al. Amygdaloid sclerosis in temporal lobe epilepsy. Ann Neurol 1993;33:622-31.

7 Miller LA, McLachlan RS, Bouwer MS, et al. Amygdalar sclerosis: preoperative indicators and outcome after temporal lobectomy. J Neurol Neurosurg Psychiatry 1994;57:1099-105.

8 Bruton C. The neuropathology of temporal lobe epilepsy. New York: Oxford University Press, 1988:29-81.

9 Bronen RA, Fulbright RK, Kim JH, et al. Regional distribution of MR findings in hippocampal sclerosis. ANNR Am J Neuroradiol 1995; 16:1193-200.

10 Cook MJ, Fish DR, Shorvon SD, et al. Hippocampal volumetric and morphometric studies in frontal and temporal lobe epilepsy. Brain 1992;115:1001-15.

11 Cendes F, Andermann F, Gloor P, et al. MRI volumetric measurement of amygdala and hippocampus in temporal lobe epilepsy. Neurology 1993:43:719-25.

12 Gambardella A, Gotman J, Cendes F, et al. The relation of spike foci and of clinical seizure characteristics to different patterns of mesial temporal atrophy. Arch Neurol 1995;52:287-93.

13 Cendes F, Andermann F, Gloor P, et al. Relationship between atrophy of the amygdala and ictal fear in temporal lobe epilepsy. Brain 1994; 1 17:739-46

14 Watson C, Andermann F, Gloor P, et al. Anatomic basis of amygdaloid and hippocampal volume measurement by magnetic resonance imaging. Neurology 1992;42:1743-50.

15 Duvernoy HM. The human hippocampus: an atlas of applied anatomy. Munich: Bergmann, 1988:61-95

16 Bland JM, Altman DG. Statistical methods for assessing agreement between two methods of clinical assessment. Lancet 1986:327:307-10.

17 Watson C, Nielsen SL, Cobb C, et al. Medial temporal lobe heterotopia as a cause of increased hippocampal and amygdaloid MRI volumes. J Neuroimaging 1996;6:231-4.

18 Wolf HK, Aliashkevich AF, Blumcke I, et al. Neuronal loss and gliosis of the amygdaloid nucleus in temporal lobe epilepsy. A quantitative analysis of 70 surgical specimens. Acta Neuropathol (Berl) 1997;93:606-10.

19 Moriarty J. A volumetric MRI study of Gilles de la Tourette's syndrome. Neurology 1997;49: 410-15.

20 Szabo CA, Fox P, Rainey L. Amygdalar volumes in controls. [Abstract] Epilepsia 1998;39:96.

21 Corsellis JAN. The neuropathology of temporal lobe epilepsy. In: Williams D, ed. Modern trends in neurology. London: Butterworth, 1970:254-70.

22 Green JR, Scheetz DG. Surgery of epileptogenic lesions of the temporal lobe. Arch Neurol 1964;10:135-48

23 Jensen I, Klinken L. Temporal lobe epilepsy and neuropathology: histological findings in resected temporal lobes correlated to surgical results and clinical aspects. Acta Neurol Scand 1976;54:391-414.

24 Bower S, Vogrin S, Kilpatrick C, et al. Hippocampal damage and duration of temporal lobe epilepsy. [Letter]. Lancet 1998;352:66-7. 